\title{
What now on the CANVAS of diabetes medications with cardiovascular protection? Could metformin, pioglitazone, SGLT2 inhibitors and liraglutide complement each other to save lives?
}

\author{
ROBERT EJ RYDER, ${ }^{1}$ RALPH A DEFRONZO²
}

On June 12th 2017 during the 77th Scientific Sessions of the American Diabetes Association in San Diego, USA, the results of the CANagliflozin cardioVascular Assessment Study (CANVAS) were presented $^{1}$ and were also published at the same time in the New England Journal of Medicine. ${ }^{2}$ All of us working to treat type 2 diabetes have been waiting with great interest for these results to see to what extent they would match those of the cardiovascular outcome study with empagliflozin (EMPA-REG OUTCOME).

In previous editorials we proposed that metformin, pioglitazone, empagliflozin and liraglutide in combination could complement each other to prevent cardiovascular events and save lives in patients with type 2 diabetes at high cardiovascular risk. 3,4 We proposed that the accumulated evidence from multiple studies suggested that pioglitazone probably exerts its beneficial effects by slowing down, or even reversing, the atherosclerotic process, whereas empagliflozin seemed to reduce cardiovascular deaths and heart failure by an entirely different, more haemodynamic, mechanism as well as perhaps by increasing circulating ketone bodies, providing the failing myocardium with a more efficient fuel source. ${ }^{3-5}$ We proposed that liraglutide, by reducing cardiovascular outcomes but, in contrast to empaglifozin, not heart failure, seemed to exert its effect through mechanisms different from those of both pioglitazone and empagliflozin. ${ }^{4,5} \mathrm{We}$ noted emerging evidence that sodium glucose transporter 2 (SGLT2) inhibitors might mitigate the fluid retention associated with pioglitazone, ${ }^{6}$ raising the possibility that pioglitazone and empagliflozin might complement each other, not only in reducing cardiovascular risk, but also in reducing side effects related to fluid retention. ${ }^{4,5}$ We pointed to the evidence that the early use of triple therapy combination of metformin, pioglitazone

${ }^{1}$ City Hospital, Birmingham, UK

${ }^{2}$ University of Texas Health Science Center, San Antonio, Texas, USA

Address for correspondence: Dr Bob Ryder

Diabetes and Endocrine Unit, City Hospital, Dudley Road, Birmingham B18 7QH, UK

E-mail: bob.ryder@nhs.net

Br J Diabetes 2017;17:89-92

http://dx.doi.org/10.15277/bjd.2017.136 and a GLP-1 receptor agonist achieved lower $\mathrm{HbA}_{1 \mathrm{c}}$, weight loss and much less hypoglycaemia compared with the traditional approach of sequential escalation through metformin, sulphonylurea and insulin, which was associated with significant weight gain. ${ }^{7}$ We also noted that the SUSTAIN 6 trial provided further evidence of cardiovascular benefit from long-acting GLP-1 receptor agonists. ${ }^{5}$ Of note, the GLP-1 receptor agonist used in SUSTAIN 6 was similar to that used in LEADER and closely resembles native GLP-1. In contrast, exenatide, which was employed in the EXSCEL trial, ${ }^{8}$ differs significantly in structure from native GLP-1 and seems to have failed to demonstrate the same level of cardiovascular protection. ${ }^{8}$ This raises questions about whether GLP-1 receptor agonists will vary in the extent to which they reduce cardiovascular events in high risk diabetic patients.

In line with previous cardiovascular outcome studies, CANVAS studied patients at high cardiovascular risk and assessed, as its primary outcome, three-point Major Adverse Cardiovascular Events (3-point MACE: cardiovascular death, non-fatal myocardial infarction and non-fatal stroke).1,2 Similar to the presentation of EMPA-REG OUTCOME in $2015^{3}$ and LEADER in 2016,4 the CANVAS findings shown in Figure $1 a^{1}$ were once again greeted with loud applause in the packed auditorium. The 14\% reduction in 3-point MACE (HR $0.86(95 \% \mathrm{Cl} 0.75$ to 0.97$))^{1,2}$ was almost identical to that from EMPA-REG OUTCOME (HR $0.86(95 \% \mathrm{Cl} 0.74$ to 0.99$)) .{ }^{9}$ Furthermore the $33 \%$ reduction in hospitalisation for heart failure (HR $0.67(95 \% \mathrm{Cl} 0.52$ to $0.87)$ ) in CANVAS 1,2 was similar to the $35 \%$ reduction found in EMPA-REG OUTCOME (HR 0.65 (95\% CI 0.50 to 0.85$)$ ). ${ }^{9}$ The similarity of the hospitalisation for heart failure curves (Figure 2), including the immediate separation between placebo versus canagliflozin and empagliflozin treatment groups suggests that the haemodynamic benefits proposed for empagliflozin ${ }^{3}$ also are seen with canaglifozin.

Given the similarities of the results from CANVAS and EMPA REG OUTCOME can we assume that the cardiovascular benefits of the two SGLT2 inhibitors are the same - representing a class effect? Professor David Matthews, summing up the presentation of the CANVAS study in San Diego ${ }^{1}$ showed the main outcomes of CANVAS and EMPA-REG OUTCOME on the same slide (Figure 3) and suggested that the results from the two trials were broadly in 
Figure 1. Side by side comparison of the effects of canagliflozin (CANVAS) and empagliflozin (EMPA-REG OUTCOME) on the cumulative incidence of 3 point major adverse cardiovascular events (MACE)

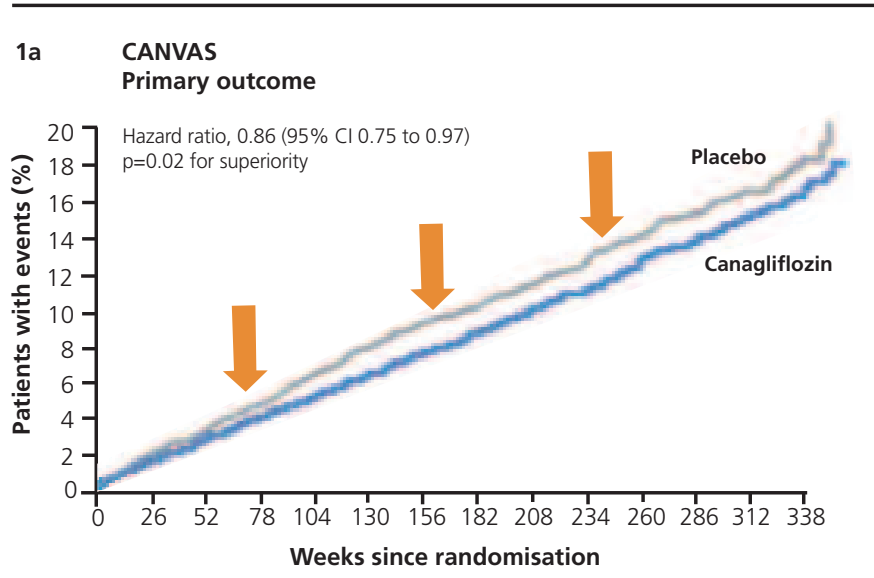

\section{EMPA-REG \\ Primary outcome}

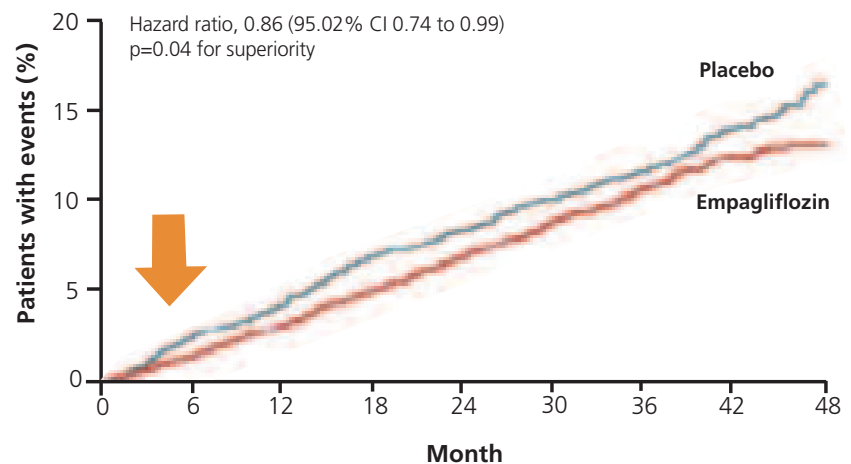

Hazard ratios [HR $(95 \% \mathrm{CI})]$ based on Cox regression analysis. Graphs adapted from reference 11.

Figure 2. Side by side comparison of the effect of canagliflozin (CANVAS) and empagliflozin (EMPA-REG OUTCOME) on cumulative incidence of hospitalisation for heart failure
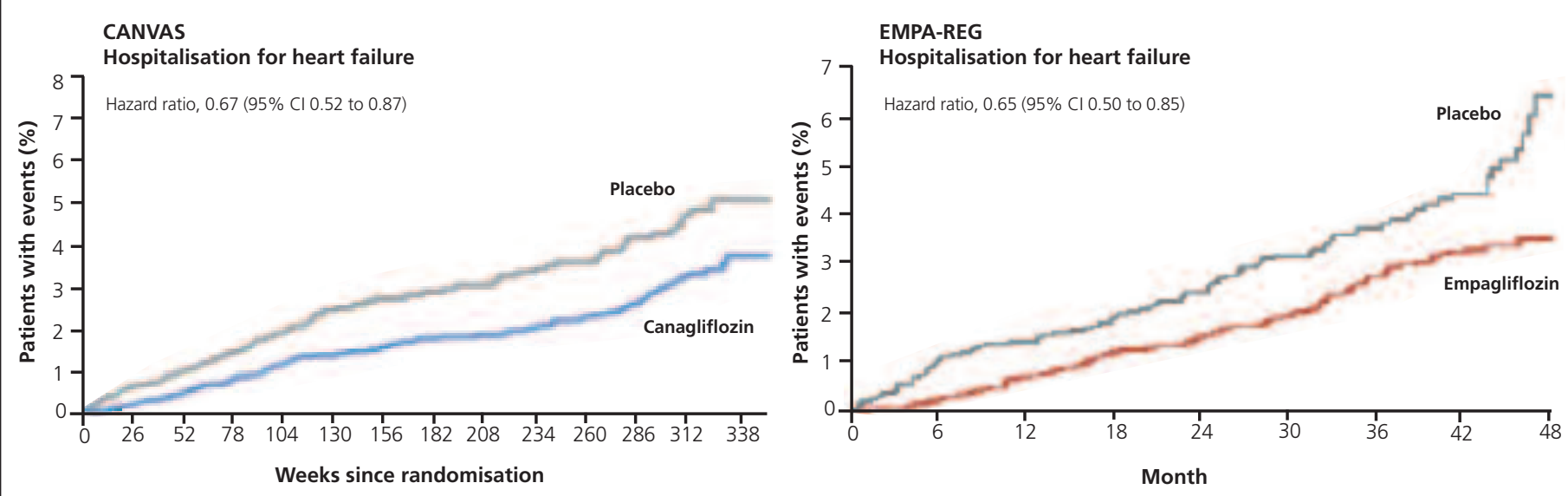

Hazard ratios [HR $(95 \% \mathrm{Cl})]$ based on Cox regression analysis. Graphs adapted from reference 11.

agreement. Nevertheless, it should be noted that although the results of 3-point MACE $(H R=0.86)$ are almost identical, the contribution of the individual components of 3-point MACE are different. Cardiovascular death was reduced to a greater extent in EMPA-REG OUTCOME (Figure 3) compared to CANVAS. Further, the effect on cardiovascular death in EMPA-REG OUTCOME was highly statistically significant $(H R=0.62, p<0.001)$ (Figure 3$)$, whereas none of the individual components of the 3-point MACE in CANVAS achieved statistical significance (Figure 3). There was a non-significant decrease in myocardial infarction in EMPA-REG OUTCOME which was offset by a non-significant increase in stroke (Figure 3). Thus, on the surface it appears as though the effect of canagliflozin and empagliflozin on the individual components of 3-point MACE may have differed in CANVAS and EMPA REG OUTCOME, although subgroup analy- ses are known to be hazardous.

If we postulate that the haemodynamic effects of empaglifozin seen in EMPA-REG are also occurring with canaglifozin in CANVAS, as suggested by the similarity of the data on hospitalisation for heart failure between the two studies (Figure 2), the uniformity of reduction of individual components of 3-point MACE in CANVAS raises the possibility that the beneficial effects of the SGLT2 inhibitors are not necessarily confined to haemodynamic benefits. It is difficult to see how haemodynamic benefits alone would reduce myocardial infarction and stroke. Thus, there may well be other factors at play and in this context it is noteworthy that SGLT2 inhibitors do reduce more standard cardiovascular risk factors such as blood pressure and weight. ${ }^{10}$

Professor Cliff Bailey in an independent commentary on the results of CANVAS, ${ }^{11}$ pointed out that the two curves of 3-point 
Figure 3. Comparison of the effects of canagliflozin (CANVAS) and empagliflozin (EMPA-REG OUTCOME) on the key outcomes in the CANVAS programme and the EMPA-REG OUTCOME. Hazard ratios [HR $(95 \% \mathrm{CI})$ ] based on Cox regression analysis

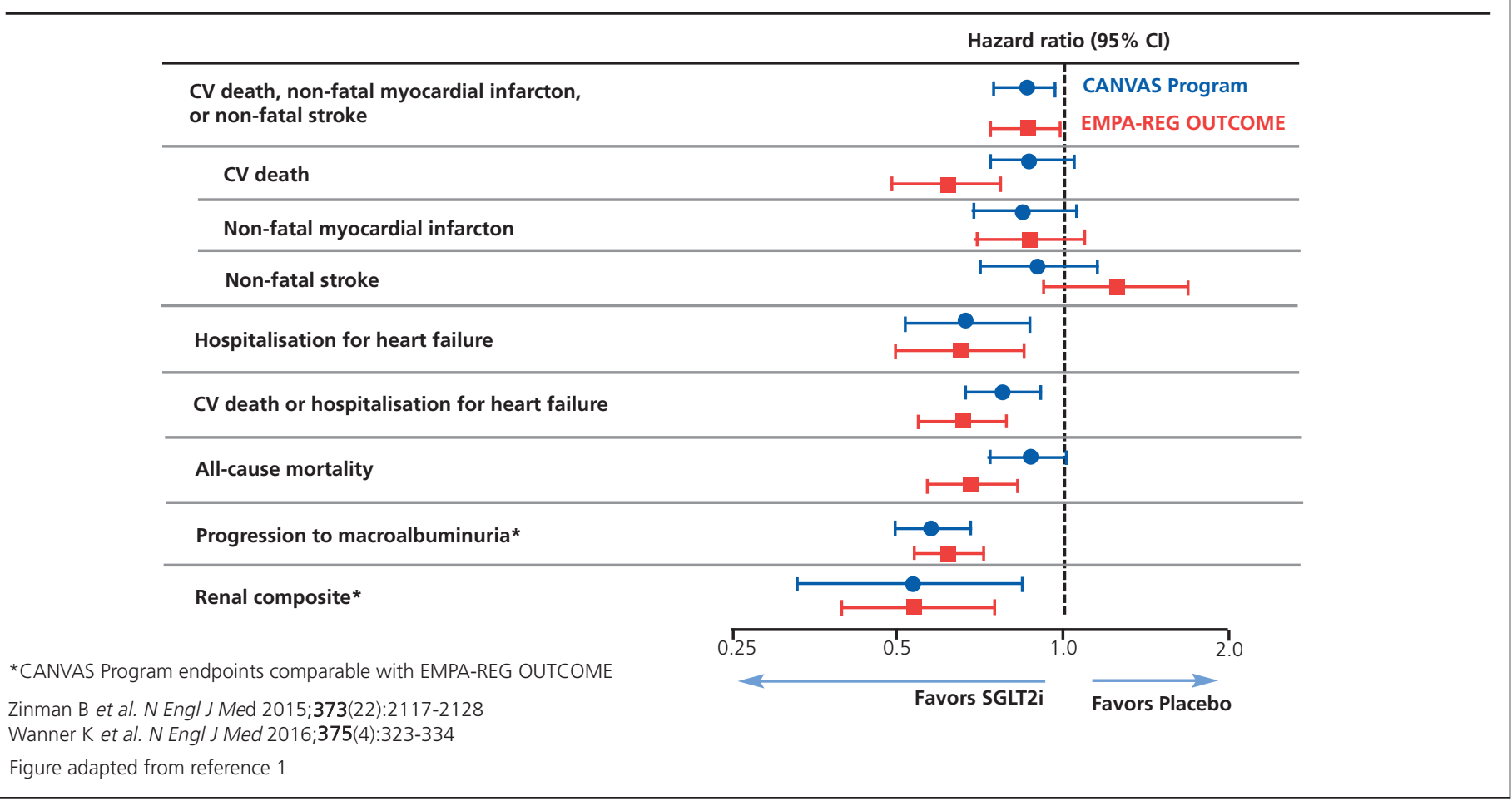

MACE do seem to separate differently in the two trials (Figure 1), occurring more slowly in CANVAS; whereas the separation is more immediate in EMPA-REG OUTCOME. It may be that these differences are due to differences in the study populations and the play of chance. However, it is also possible that, while some effects of the two agents are similar, they also have differing effects. There was an increase in amputations in the canagliflozintreated group compared to the placebo-treated group (HR 1.97 (95\% Cl 1.41 to 2.75$)$ ). 1,2 Of these, $71 \%$ of these were minor amputations (toe or metatarsal) but $29 \%$ were major amputations (ankle, below-knee and above-knee).1,2 The hazard ratio was especially increased in those with a history of previous amputation (HR 20.9 (95\% Cl 14.2 to 30.8)) but was also increased in those with a history pf peripheral vascular disease without amputation (HR 3.1 (95\% Cl 2.2 to 4.5)). 1,2 This increase in amputations in CANVAS remains unexplained. The European Medicines Agency (EMA) response to the possibility of an increase in amputations in association with canaglifozin has been to advise doctors and patients to be alert to the possibility of this risk with all the SGLT2 inhibitors. ${ }^{12}$ The EMA notes that an increase in lower limb amputations has not been seen in studies with other medicines in the same class, dapagliflozin and empagliflozin, but that data available to date are limited and the risk may also apply to these other medicines. ${ }^{12}$ Given the data, it would certainly seem prudent to consider avoiding canagliflozin in patients with previous amputations, with peripheral artery disease and indeed in those with previous foot complications. In line with the EMA guidance ${ }^{12}$ there might also be a case for being cautious about all agents in the SGLT2 inhibitor class in such patients.

When considering whether there are real differences between empaglifozin and canaglifozin that can be inferred by comparing CANVAS with EMPA-REG OUTCOME, we need to be mindful that the populations being studied were different. In EMPA-REG OUTCOME the population was almost entirely secondary prevention - patients who previously had a cardiovascular event. ${ }^{9}$ Only $65 \%$ of the patients in CANVAS represented secondary prevention with 35\% primary prevention. ${ }^{1,2}$ As pointed out by Professor David Matthews in his summary of CANVAS, ${ }^{1}$ the comparison of the data between EMPA-REG OUTCOME and CANVAS is complicated by differences in populations being studied, trial designs, analytical approaches and drug effects; thus, comparisons are hazardous, subject to bias and confounded by multiple uncontrolled factors. ${ }^{1}$ While being mindful of the differences in outcomes discussed with regard to Figure 3, we agree with David Matthews' overall suggestion that the results from the two studies are broadly in agreement.

Now that the results of CANVAS have been added to those of PROactive, EMPA-REG OUTCOME, LEADER and SUSTAIN-6, we can perhaps speculate, from the points made above and in our previous editorials, ${ }^{3-5}$ that the combination of metformin, pioglitazone, an SGLT2 inhibitor (in particular, empaglifozin and canaglifozin) and liraglutide appears to be the optimum cocktail of medications for improving both glycaemic control and cardiovascular outcomes for people with type 2 diabetes at high cardiovascular risk. Further, the evidence we have today suggests 


\section{Key messages}

- In previous editorials following EMPA-REG OUTCOME and LEADER we concluded that pioglitazone, empagliflozin and liraglutide might complement each other to prevent cardiovascular events and save lives by different mechanisms

- The CANVAS study has shown that canagliflozin reduced 3-point MACE (cardiovascular death, non-fatal myocardial infarction and non-fatal stroke) by exactly the same amount as empagliflozin in the EMPA-REG OUTCOME trial. Similarly, the impact on hospitalisation for heart failure was almost identical in CANVAS and EMPA-REG OUTCOME. This suggests similar haemodynamic effects for the 2 SGLT2 inhibitors in the two trials

- Differences between the individual components of 3-point MACE in the two trials, however, have raised the possibility of effects over and above haemodynamic effects. An increase in amputations in the CANVAS trial with canagliflozin is unexplained and it is noted that the European Medicines Agency (EMA) has urged caution as a result for all SGLT2 inhibitors, but caution should be especially exercised for the class (in particular canagliflozin) in patients with previous foot complications

- The combination of metformin, pioglitazone, an SGLT2 inhibitor (in particular empagliflozin or canagliflozin) and liraglutide now appears to be the optimum cocktail of medications for improving both glycaemic control and cardiovascular outcome for people with type 2 diabetes with high cardiovascular risk. The evidence suggests that these agents in combination could complement each other to prevent cardiovascular events and save lives

that the agents in this combination may complement each other to prevent cardiovascular events and save lives, although this remains to be proven by randomised, prospective cardiovascular outcome trials. Nevertheless, we would propose caution with regard to the use of SGLT2 inhibitors (in particular canagliflozin) in those with a history of amputation, peripheral arterial disease or previous foot complications.
Conflict of interest REJR has received speaker fees, and/or consultancy fees and/or educational sponsorships from AstraZeneca, BioQuest, GI Dynamics, Janssen, Novo Nordisk, Sanofi-Aventis and Takeda.

RAD: Advisory Board: AstraZeneca, Novo Nordisk, Janssen, Lexicon, Boehringer-Ingelheim; Research Support: Bristol-Myers Squibb, BoehringerIngelheim, Takeda, AstraZeneca; Speaker's Bureau: Novo Nordisk, AstraZeneca

\section{References}

1. The CANVAS Program (CANagliflozin cardioVascular Assessment Study). The slide set used at the presentation of the CANVAS study results on June 12th 2017 during the 77th Scientific Sessions of the American Diabetes Association in San Diego, USA. http://www.georgeinstitute.org/sites/default/ files/canvas-study-results-ada-2017.pdf (accessed 3 July 2017)

2. Neal B, Perkovic $V$, Mahaffey KW, et al. Canagliflozin and cardiovascular and renal events in type 2 diabetes. N Engl J Med 2017. https://doi.org/10.1056/NEJMoa1611925. [Epub ahead of print]

3. Ryder REJ, DeFronzo RA. Diabetes medications with cardiovascular protection in the wake of EMPA-REG OUTCOMETM: the optimal combination may be metformin, pioglitazone and empagliflozin. Br J Diabetes Vasc Dis 2015;15:151-4. http://dx.doi.org/10.15277/bjdvd.2015.045

4. Ryder REJ, DeFronzo RA. Diabetes medications with cardiovascular protection - what now after LEADER®? Could metformin, pioglitazone, empagliflozin and liraglutide complement each other to save lives? $\mathrm{Br} J$ Diabetes 2016;16:103-06. http://dx.doi.org/10.15277/bjd.2016.096

5. Abdul-Ghani M, DeFronzo RA, Del Prato S, Chilton R, Singh R, Ryder REJ. Cardiovascular disease and type 2 diabetes: has the dawn of a new era arrived? Diabetes Care 2017;40(7):813-20. https://doi.org/10.2337/dc16-2736.

6. Gautam A, Agrawal PK, Prakash P, Hazra DK. Pioglitazone associated pedal edema resolved by adding sodium glucose co-transporter 2 in-hibitor. ADA 76th Scientific Sessions 2016. Late Breaking Poster Session, Poster 140-LB. Abstract available at http://www.abstractsonline.com/pp8/ \#!/4008/presentation/44543 (accessed 27 July 2017)

7. Abdul-Ghani MA, Puckett C, Triplitt C, et al. Initial combination therapy with metformin, pioglitazone and exenatide is more effective than sequential add-on therapy in subjects with new-onset diabetes. Results from the Efficacy and Durability of Initial Combination Therapy for Type 2 Diabetes (EDICT): a randomized trial. Diabetes Obes Metab 2015; 17:268-75. http://dx.doi.org/10.1111/dom.12417

8. AstraZeneca. Bydureon EXSCEL trial meets primary safety objective in type-2 diabetes patients at wide range of cardiovascular risk. Press release 23 May 2017. https://www. astrazeneca.com/media-centre/press-releases/2017/bydureon-exscel-trial-meets-primary-safety-objective-in-type2-diabetes-patients-at-wide-range-of-cardiovascular-risk-23052017.html (accessed 7 July 2017)

9. Zinman B, Wanner C, Lachin JM, et al. Empagliflozin, cardiovascular outcomes, and mortality in type 2 diabetes. N Engl J Med 2015;373:211728. http://dx. doi.org/10.1056/NEJMoa1504720

10. Tahrani AA, Barnett AH, Bailey CJ. SGLT inhibitors in management of diabetes. Lancet Diabetes Endocrinol 2013;1(2):140-51. https://doi.org/10.1016/S2213-8587(13)70050-0. Epub 2013 Aug 13.

11. Bailey CJ. CANVAS Program Independent Commentary. The slide set used for the independent commentary after the presentation of the CANVAS study results on June 12th 2017 during the 77th Scientific Sessions of the American Diabetes Association in San Diego, USA. http://www.georgeinstitute.org/sites/default/files/canvas-independentcommentary-ada.pdf (accessed 3 July 2017)

12. European Medicines Agency. SGLT2 inhibitors: information on potential risk of toe amputation to be included in prescribing information. 20 April 2017. http://www.ema.europa.eu/ema/index.jsp?curl=pages/medicines/ human/referrals/SGLT2_inhibitors_(previously_Canagliflozin)/human_referral_prac_00 0059.jsp\&mid=WC0b01ac05805c516f (accessed 3 July 2017) 